\title{
Propuesta metodológica para el conocimiento de la educación ambiental de forma transdisciplinar
}

\section{Methodological proposal for the knowledge of environmental education in a transdisciplinary way}

\author{
Gabriela Regalado Pineda ${ }^{1}$
}

Carmen Ernestina López Rubio ${ }^{2}$

\begin{abstract}
Resumen
En un modelo por competencias, tanto docentes como alumnos deben contribuir a la creación de ambientes que propicien una convivencia armoniosa de la sociedad. En este trabajo se presenta una propuesta metodológica para abordar problemáticas ambientales de forma transdisciplinar en sexto semestre de bachillerato de la Universidad de Guadalajara. La propuesta tiene como objetivo particular los contenidos relacionados con una educación ambiental, para que el alumno logre comprender, proponer y participar en alternativas de solución a los problemas de su entorno.
\end{abstract}

\section{Palabras clave}

Transdisciplinariedad, interdisciplinariedad, proyecto, sustentable.

\section{Abstract}

In a model based on competences, both teachers and students must contribute to the creation of environments conducive to a harmonious

1 Gabriela Regalado Pineda. Pertenece el equipo de formadores de la Dirección de Formación Docente e Investigación de la Universidad de Guadalajara, Jalisco, México. Es Profesora de asignatura B de la Escuela Preparatoria Núm. 15. Correo electrónico: gabyregalado@hotmail.com

ID: http://orcid.org/0000-0002-9025-3946

2 Carmen Ernestina López Rubio. Profesora de asignatura B de la Escuela Preparatoria No. 15 de la Universidad de Guadalajara, Jalisco, México. Pertenece al equipo de formadores de la Dirección de Formación Docente e Investigación de la Universidad de Guadalajara. Correo electrónico: krmenzl@yahoo.com.mx

ID: http://orcid.org/0000-0002-0999-983X 
coexistence of society. In this paper, we present a methodological proposal to address environmental problems in a transdisciplinary way in the sixth semester of the baccalaureate of the University of Guadalajara. The proposal has as a particular objective the contents related to environmental education, so that the student can understand, propose and participate in alternative solutions to the problems of their environment.

\section{Keywords}

Transdisciplinarity, interdisciplinary, project, sustainable.

\section{Introducción}

Es común la problemática de planear de forma desarticulada proyectos que inciden en el aprendizaje. En este trabajo se presenta una propuesta sobre el diseño transversal que logre la integración de saberes que permitan la adquisición de diferentes conocimientos para que el alumno encuentre soluciones a los problemas ambientales de su comunidad con base en una conciencia ética.

Propuesta que tiene su origen en el ciclo 2009B y que comenzó con un trabajo interdisciplinar, se convirtió en un multidisciplinar hasta pretender llegar a un transdisciplinar que, con la articulación de la Unidades de Aprendizaje de 6o semestre de Geografía y cuidado del entorno, Análisis económico y Habilidad verbal, se logre construir en el alumno una formación de respeto hacia al medio ambiente de su entorno.

Por lo tanto, uno de los objetivos de este trabajo es la articulación de contenidos temáticos; la propuesta considera como eje central la materia de Geografía y cuidado del entorno de tal forma, que el conocimiento adquirido refleje en el alumno en su habilidad para la elaboración de proyectos, pero sobre todo, el desarrollo de competencias que lo lleven a tomar conciencia sobre una cultura ambiental, para la realización de acciones encaminadas a entender la problemática y tomar decisiones responsables para darles solución.

\section{Antecedentes}

Es un proyecto que nace con la necesidad de simplificar al alumno la realización de proyectos para ser evaluado, ya que los docentes involucrados trabajaban la pluridisciplinariedad, es decir, cada uno planeaba su propio proyecto y lo abordaba implícitamente con varias disciplinas a la vez. 
El proyecto de la Unidad de Aprendizaje de Habilidad Verbal consistía en hacer un ensayo sobre problemas del medio ambiente, contenidos de forma que se abordaban en Geografía y cuidado del entorno; Geografía se les solicitaba un proyecto sustentable en el que evaluaba los argumentos y elementos de forma; Economía evaluaba con un museo en el cual tenían que elaborar el guión museográfico y museológico.

\section{Gráfico 1: Pluridisciplinariedad}

A pesar de que no se requería interacción entre los docentes, hubo intercambio de saberes entre éstos y así se comienza a trascender los límites de cada Unidad involucrada.

Con base en esa pluridisciplinariedad, que es estudiar un objeto con una solo disciplina por varias disciplinas a la vez (Nicolescu, 1996), se hizo un trabajo de planeación interdisciplinar en la que las tres Unidades de Aprendizaje solicitaron un proyecto de carácter mixto, que consistió en una misma Revista que como producto integrador.

En esta ocasión las tres disciplinas, cada una desde su área iban conformando el conocimiento que se reflejaría en el producto integrador; se transfieren conocimientos de una a otra disciplina, es decir, los docentes abordan temas de cualquiera de las tres para abonar al conocimiento.

\section{Gráfico 2: Interdisciplinariedad}

Esta forma de trabajo se llevó a cabo hasta el 2016A, periodo que dio como resultado varios proyectos integradores como Revista especializada, Programa de radio de sustentabilidad y economía, Museo de sustentabilidad y economía y Proyecto sustentable. En la siguiente tabla se presenta el objetivo de aprendizaje y ciclo en el que se trabajaron.

\section{Tabla 1: Proyectos transversales interdisciplinares}

Con este trabajo transversal se logró una evaluación interdisciplinar de forma colegiada; involucrar a los alumnos en el trabajo colaborativo y activo en proyectos de sustentabilidad que los compromete con su defensa; poner en práctica dichos proyectos y compartir la experiencia y que recibir retroalimentación en el momento.

Un logro importante y relevante fue el presentado por una exalumna y ahora alumna de la Licenciatura en Urbanística y Medio Ambiente de la Universidad de Guadalajara, que presentó el proyecto de sustentabilidad realizado como producto interdisciplinar en la prepa, logró que se lo aceptaran cuando realizó trámites a dicha licenciatura como proyecto de admisión. 
Sin embargo, con la experiencia docente que se fue adquiriendo con esta forma de trabajo, se observa que los alumnos se concentran en los contenidos temáticos de los proyectos a presentar, pero no manifiestan una conciencia hacia los temas ambientales. Entonces se hizo hacer modificaciones de nueva cuenta a la planeación transversal, ahora con un modelo transdisciplinar que dé respuesta al cómo lograr que el alumno tome decisiones que privilegien la preservación del ambiente a lo largo de su vida y no sólo en un producto integrador.

\section{Propuesta metodológica}

Con base en estos antecedentes se presenta la propuesta que además de lograr la interdisciplinariedad, contribuye a acciones más conscientes que además de favorecer la educación ambiental como lo establece la competencia genérica 11 (SEP, Acuerdo 444), permita lograr una conexión de saberes que favorezca la articulación de distintos conocimientos en la apropiación de problemas del entorno y la formación de una cultura ambiental que responda a sus exigencias sociales, educativas y ecológicas.

El diseño de esta propuesta metodológica tiene como base el modelo de transdiciplinariedad que propone la construcción conjunta del conocimiento de las diversas disciplinas que intervienen así como una cultura ambiental a partir de un proyecto de educación ambiental donde se integre lo propuesto por Caride (2001), Luhman (2002) y Nicolescu (2006).

Caride (2001) establece que la educación ambiental va más allá de las relaciones pedagógicas; ésta significa una preparación para que el alumno sea capaz de generar cambios que reafirmen un desarrollo sustentable, así como generar una conciencia que lleve a la solución de los problemas actuales; ésta debe propiciar un cambio de actitudes como lo es la participación en la creación de acciones adecuadas con su entorno.

Afirma que la educación ambiental debe configurarse integrando conocimiento, pensamiento y acciones. Acciones que lleven a la toma de decisiones y valoración de sus consecuencias tanto personales como colectivas.

Al respecto, Luhman (2002) establece que las decisiones son la parte inconsciente, impregnadas de las estructuras latentes de la persona, esto es, los valores, las ideas y creencias de los alumnos, en cuanto a su forma de ver y apreciar la sustentabilidad ecológica.

La parte visible de las decisiones son las acciones y para que haya una acción debe haber un desequilibrio, en este contexto, es aquello con lo 
que el alumno no está conforme respecto a su medio ambiente o considera puede ser mejorado.

En ese sentido, Nicolescu (2006) afirma que el prefijo "trans" del concepto de transdisciplinariedad, denota algo que va a través, entre y más allá que reclama una ampliación de la conciencia orientada hacia los sentimientos, autenticidad, solidaridad, generosidad, cooperación al sentirse como miembro de una colectividad.

La transdisciplinariedad es una postura que va más allá de lo disciplinario, pluri e interdisciplinario ya que constituye una visión del mundo a través de tres pilares que determinan su metodología:

1) La existencia, en la Naturaleza y en nuestro conocimiento de la Naturaleza, de diferentes niveles de Realidad. El conjunto de los niveles de la realidad constituye el proyecto transdisciplinar. Gráfico 3: Pilar 1

2) El paso de un nivel de realidad a otro se efectúa por la lógica del tercero incluido. Lo que puede ser respondido con sólo dos aristas en un primer nivel, se modifica al cambiar de nivel de realidad. Gráfico 4: Pilar 2

3) La estructura del ensamblaje de los niveles de Realidad es una estructura compleja: cada nivel es tal porque los otros niveles existen a la vez. Los diferentes enfoques de la realidad se entretejen conformado una sola. Gráfico 5: Pilar 3

Los tres pilares de la transversalidad se interpretan en la siguiente tabla con base en la propuesta.

Tabla 2: ejes que determinan la metodología de la propuesta. De ahí que la aportación de esta propuesta transdisciplinar no sólo proviene de abordar los contenidos económicos, de sustentabilidad y habilidad verbal de una manera conjunta y vincularlos con la realidad del alumno, sino promover la empatía, solidaridad y afectividad hacia una cultura sustentable independientemente de si carga o no con ellos en sus estructuras latentes.

\section{Gráfico 6: Transdisciplinariedad}

Las tapas para la planeación transdisciplinar es la siguiente:

Etapa 1. RECUPERAR INFORMACIÓN: Hacer una carta descriptiva de las Unidades de Aprendizaje con el fin de visualizar resultados de aprendizaje, contenidos y actividades integradoras, para poder diseñar el proyecto transversal a evaluar.

Etapa 2. FORMULACIÓN: Una vez que se obtienen las cartas descriptivas de las diferentes Unidades de Aprendizaje (Materias), se 
procede a su revisión y decidir cuál sería el eje transversal del producto integrador del proyecto.

Etapa 3. PLANEAR: Después de tomada la decisión de forma colegiada, sobre el producto integrador, se procede a planear considerando los contenidos de las Unidades de Aprendizaje (Materias) involucradas.

Etapa 4. DISEÑAR: En esta etapa del proyecto, se diseña de forma colegiada la rúbrica transdisciplinar. Ésta incluye los aspectos a evaluar de cada Unidad de Aprendizaje y Listas de Cotejo y/o Guía de Observación.

Etapa 5. INTEGRACIÓN: Esta fase se pone en marcha dentro las diferentes Unidades de Aprendizaje (Materias). Consiste en que todos los docentes involucrados hablan del mismo proyecto, la diferencia está en que cada uno profundiza en sus propios contenidos y se abordan dilemas ambientales con el propósito de que el alumno ponga en práctica la toma de decisiones.

Etapa 6. EVALUACIÓN TRANSDISCIPLINAR: Esta última etapa consiste en la evaluación colegiada de las exposiciones de los alumnos y se lleva a cabo de la siguiente manera:

a) Se asigna fecha y hora a cada equipo para la presentación de su proyecto.

b) El equipo presenta el proyecto frente a todos los docentes involucrados.

c) Cada integrante registra una autoevaluación sobre su proyecto y presentación del mismo.

d) Cada uno de los docentes hace preguntas sobre lo expuesto, retroalimenta y califica

e) Se hace la sumatoria de los diferentes indicadores de la rúbrica y esa sumatoria es la calificación que se le registra a los integrantes del equipo en todas las Unidades de Aprendizaje (Materias).

Según Nicolescu (2009), en la concepción transdisciplinaria de la educación existe una trans-relación que vincula los cuatro pilares del nuevo sistema educativo propuestos por el Informe Delors, aprender a conocer, aprender a hacer, aprender a convivir y aprender a ser. Esa trans-relación reposa sobre los cuatro pilares; si uno solo de estos pilares se derrumba, todo se derrumba. Una educación viable sólo puede ser una educación integral del hombre, una educación que se dirige a la totalidad abierta del ser humano y no a uno solo de sus componentes. 


\section{Conclusiones}

La transversalidad es importante en la elaboración de proyectos ya que permite la intervención de contenidos de aprendizaje de varias disciplinas, en torno a la solución de un problema y así, lograr un análisis crítico que contribuye a un conocimiento significativo.

Es tiempo de dejar de lado el conocimiento fragmentado y enfrentar los problemas de nuestra realidad con una metodología transdisciplinar, la cual no sólo transfiere conocimientos de una a otra disciplina, sino que involucra valores y virtudes para un mejor entorno.

Para llegar a un trabajo transversal de tipo transdisciplinar, es necesario que se trabaje lo disciplinar y pluridisciplinar, sin absolutizar esta distinción. En este trabajo se presentan de forma separada sólo para explicar la evolución del trabajo académico realizado.

Para la construcción del pensamiento, es necesario un trabajo de planeación transdisciplinar que permita al alumno tener un nuevo tipo de pensamiento y de acción social.

Esta propuesta metodológica logra, además de un análisis crítico de los problemas ambientales, el trabajo colegiado de docentes y alumnos en la búsqueda de soluciones a la sustentabilidad del ambiente.

\section{Referencias}

Caride, Gómez J. A. (2001). La educación ambiental en el desarrollo humano: horizontes para la sustentabilidad ecológica y la responsabilidad social. Disponible en:

http://www.mapama.gob.es/es/ceneam/articulos-de-opinion/200110caride tcm7-141789.pdf

Luhmann, N. (1994) ¿Cómo se pueden observar estructuras latentes? En Contribuciones al constructivismo. (pp. 60 - 72) Barcelona, Ed. Gedisa.

Nicolescu , Basarab (s/f). La transdisciplinariedad. Manifiesto. Ediciones Du Rocher. Disponible en: http://www.ceuarkos.com/manifiesto.pdf Secretaría de Educación Pública. (2008). Acuerdo 444. Diario Oficial de la Federación. Disponible en:

http://www.copeems.mx/images/pdf/acuerdo\%20numero\%20442 $\% 20$ snb.pdf

Sistema de Educación Media Superior. (2009). Bachillerato General por Competencias del Sistema de Guadalajara, México: Universidad de Guadalajara. Recuperado de http://www.sems.udg.mx/sites/default/files/BGC/BGCUDG Documento base evaluado COPEEMS.pdf 
RECIE. Revista Electrónica Científica de Investigación Educativa

Vol. 4, núm. 2, enero-diciembre 2019, pp. 1007-1017.

\section{Tablas y cuadros}

Tabla 1: Proyectos transversales Interdisciplinares

\begin{tabular}{|l|l|c|}
\hline \multicolumn{1}{|c|}{$\begin{array}{c}\text { Nombre del } \\
\text { proyecto }\end{array}$} & \multicolumn{1}{|c|}{ Objetivo de Aprendizaje } & Ciclo \\
\hline $\begin{array}{l}\text { Revista } \\
\text { Especializada }\end{array}$ & $\begin{array}{l}\text { El alumno realiza una revista de manera } \\
\text { transversal con sus unidades de aprendizaje } \\
\text { Geografía y Cuidado del entorno y Análisis } \\
\text { económico, a través de la cual pone de } \\
\text { manifiesto un correcto uso de la redacción y } \\
\text { ortografía. }\end{array}$ & 2013 A y B \\
\hline $\begin{array}{l}\text { Proyecto } \\
\text { sustentable }\end{array}$ & $\begin{array}{l}\text { El alumno construye argumentos } \\
\text { pertinentes, en el proyecto de } \\
\text { sustentabilidad que presenta de forma } \\
\text { transversal con las Unidades de Aprendizaje } \\
\text { de Geografía y cuidado del entorno y Análisis } \\
\text { económico. }\end{array}$ & 2014 A y B \\
\hline $\begin{array}{l}\text { Museo de } \\
\text { sustentabilidad } \\
\text { y economía }\end{array}$ & $\begin{array}{l}\text { El alumno relacionará los principios } \\
\text { macroeconómicos y el desarrollo } \\
\text { sustentable, proyectándolos en un museo, en } \\
\text { el cual pone de manifiesto un correcto uso de } \\
\text { la redacción y ortografía. }\end{array}$ & 2015 A y B \\
\hline $\begin{array}{l}\text { Programa de } \\
\text { radio de } \\
\text { sustentabilidad } \\
\text { y economía }\end{array}$ & $\begin{array}{l}\text { El alumno realiza un programa de radio de } \\
\text { sustentabilidad y economía relacionados con } \\
\text { los contenidos temáticos de Geografía y } \\
\text { Cuidado del Entorno y Análisis Económico y } \\
\text { dan evidencia de su dominio en la } \\
\text { elaboración de textos creativos y coherentes, } \\
\text { así como de la correcta expresión oral. }\end{array}$ & 2016 A y B \\
\hline
\end{tabular}

Tabla 2: Ejes que determinan la metodología de la propuesta.

\begin{tabular}{|l|l|}
\hline \multicolumn{1}{|c|}{ Nivel } & \multicolumn{1}{c|}{ Interpretación en la propuesta } \\
\hline Niveles de realidad & $\begin{array}{l}\text { El proyecto se conforma con base en los contenidos } \\
\text { temáticos de las diferentes Unidades de Aprendizaje } \\
\text { involucradas, las estructuras latentes del alumno y el } \\
\text { trabajo de concientización en clase. }\end{array}$ \\
\hline $\begin{array}{l}\text { Lógica de tercero } \\
\text { incluido }\end{array}$ & $\begin{array}{l}\text { En cada parcial hay evidencias que permiten ver que en un } \\
\text { nivel de realidad que son antagónicos, en otro nivel de } \\
\text { realidad, pueden ser complementarios. }\end{array}$ \\
\hline La complejidad & $\begin{array}{l}\text { La estructura de la totalidad de los niveles de la realidad o } \\
\text { percepción es una estructura compleja que incluye los }\end{array}$ \\
\hline
\end{tabular}

Regalado Pineda, G.; y López Rubio, C.E. 


\begin{tabular}{|l|l|}
\hline & $\begin{array}{l}\text { contenidos de las Unidades de aprendizaje que participan, } \\
\text { así como las ideas, creencias y valores de los alumnos, lo } \\
\text { que da origen a una realidad diferente. }\end{array}$ \\
\hline
\end{tabular}

\section{Gráficas e imágenes}

Gráfico 1: Pluridisciplinariedad

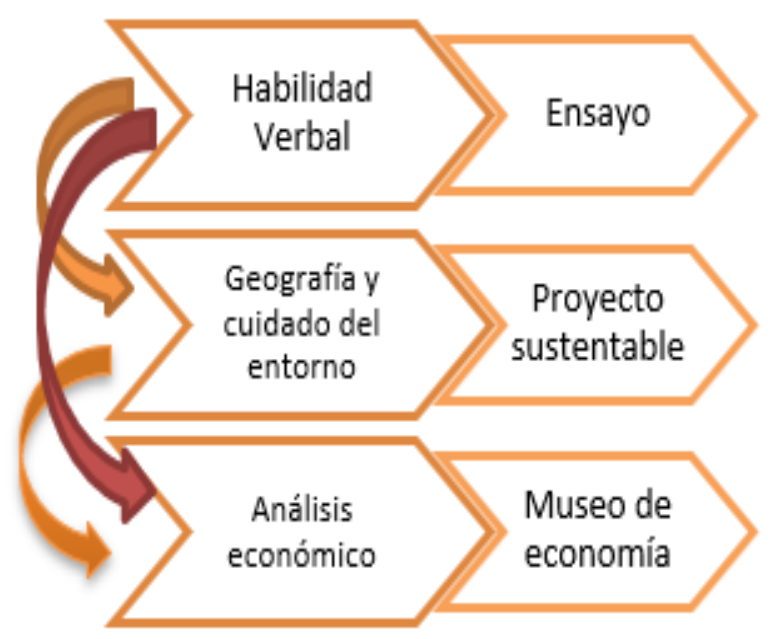

Gráfico 2: Interdisciplinariedad.

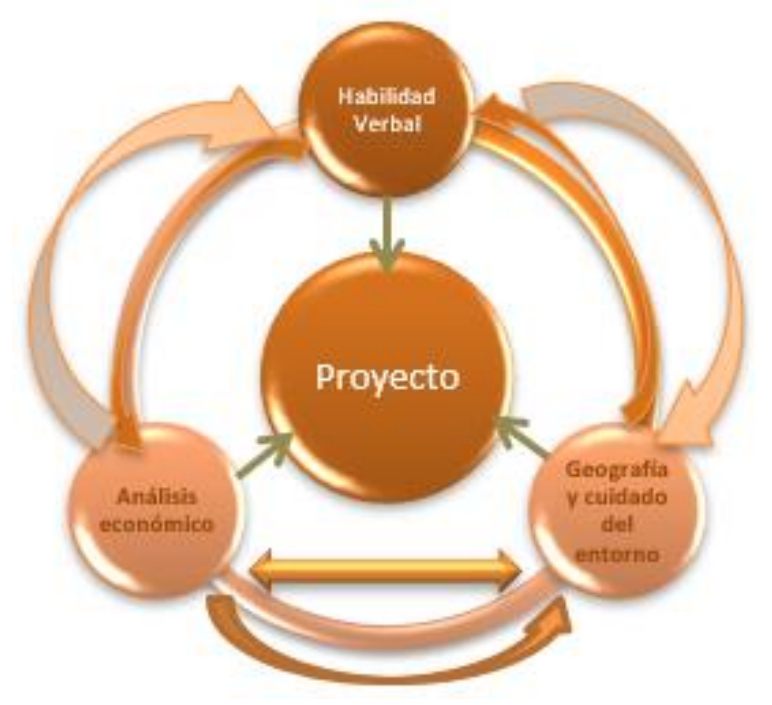

Propuesta metodológica para el conocimiento de la educación ambiental... 
RECIE. Revista Electrónica Científica de Investigación Educativa Vol. 4, núm. 2, enero-diciembre 2019, pp. 1007-1017.
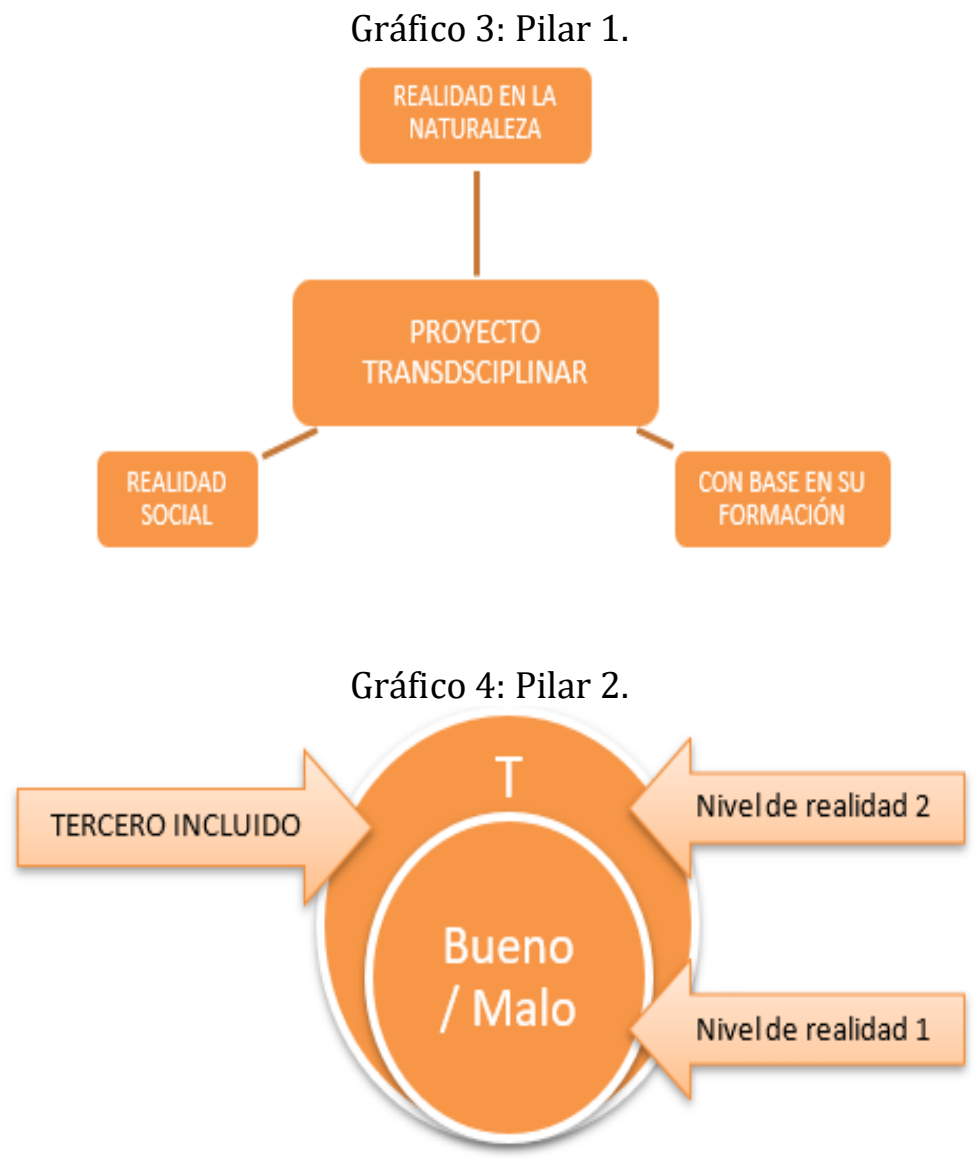

Gráfico 5: Pilar 3

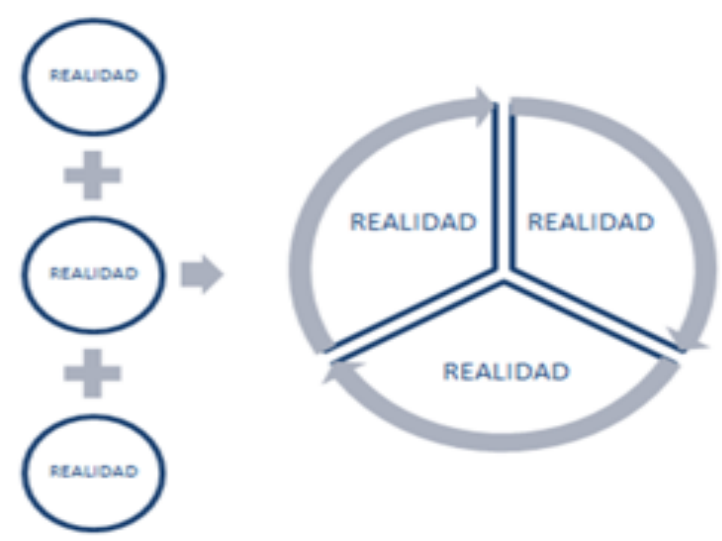

Regalado Pineda, G.; y López Rubio, C.E. 


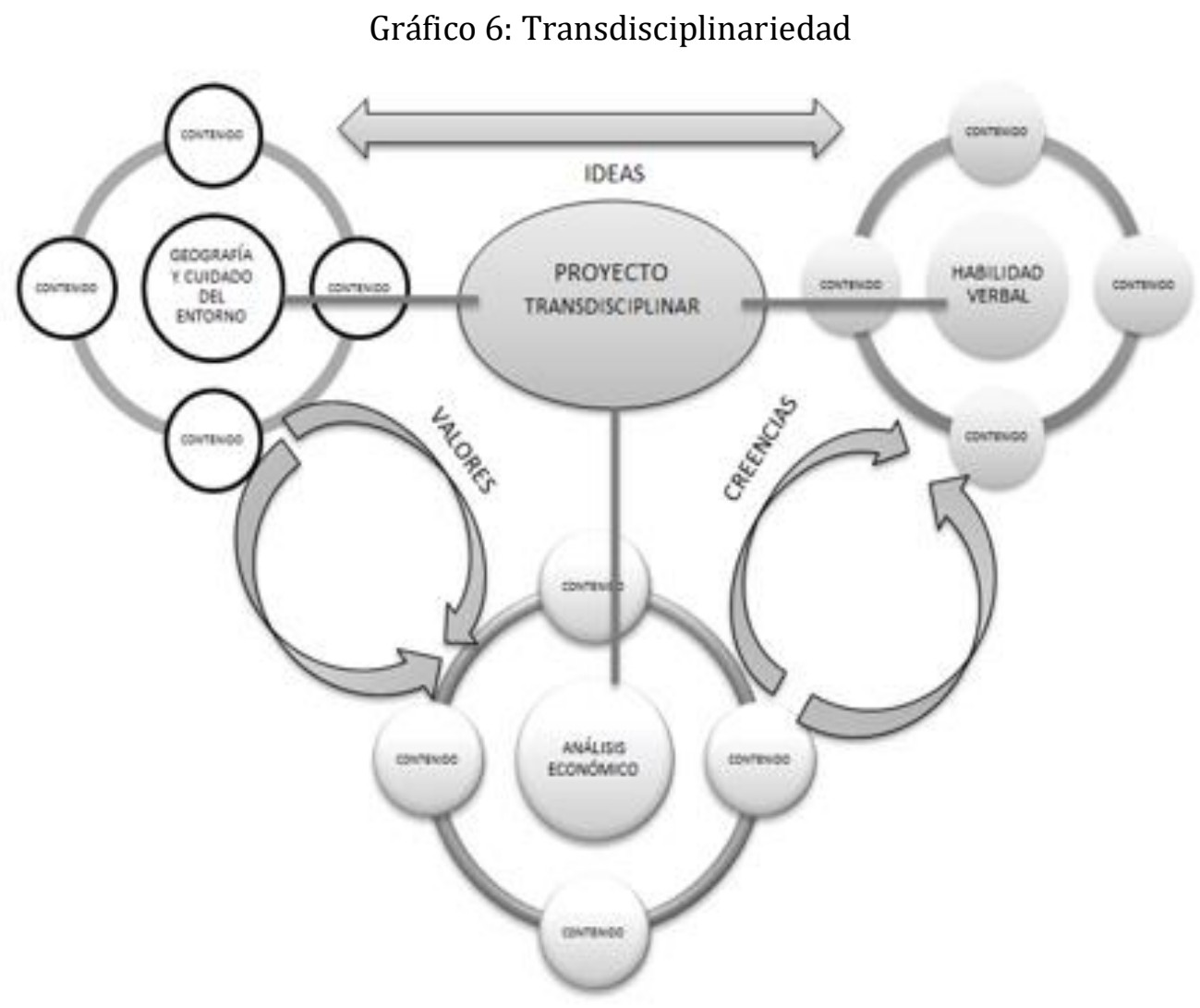

Propuesta metodológica para el conocimiento de la educación ambiental... 
RECIE. Revista Electrónica Científica de Investigación Educativa Vol. 4, núm. 2, enero-diciembre 2019, pp. 1007-1017. 